\title{
Pentoxifylline Inhibits Interleukin-2-induced Toxicity in C57BL/6 Mice but Preserves Antitumor Efficacy
}

\author{
Michael J. Edwards, ${ }^{*}$ B. Todd Heniford,“ Elizabeth A. Klar,“ Karola W. Doak, ${ }^{\star}$ and Frederick N. Miller,” \\ Departments of ${ }^{*}$ Surgery and ${ }^{\ddagger}$ Physiology, J. Graham Brown Cancer Center, University of Louisville \\ School of Medicine, Louisville, Kentucky 40292
}

\begin{abstract}
Interleukin 2 (IL-2) mediates the regression of metastatic cancer but clinical use has been limited due to associated toxicities. Tumor necrosis factor (TNF) is an important mediator of IL-2 toxicity and may have a limited role in IL-2 antitumor efficacy. Because pentoxifylline (PTXF) inhibits TNF production, we hypothesized that PTXF would ameliorate IL-2 toxicity without compromising antitumor efficacy. Four groups of female C57BL/6 mice with pulmonary metastases from a 3-methylcholanthrene-induced fibrosarcoma (MCA-105) and four groups of nontumored mice were treated every $6 \mathrm{~h}$ for $4 \mathrm{~d}$ by intraperitoneal injections of either IL-2 alone, IL-2 and PTXF, PTXF alone, or equal volumes of saline. Upon completion of therapy, we found that PTXF suppressed many of the IL-2-induced effects including TNF production, lymphocytic infiltration of multiple organs, multiple organ edema, hepatic dysfunction, leukopenia, and thrombocytopenia. Tumor response was determined $21 \mathrm{~d}$ after cessation of therapy by quantitating the number and surface area of pulmonary metastases. PTXF preserved antitumor efficacy while reducing the morbidity and mortality caused by IL-2 treatment. These data strongly support the use of PTXF in extending the therapeutic index of IL-2 in the treatment of cancer. (J. Clin. Invest. 1992. 90:637641.) Key words: endothelium • lymphocytic infiltration • therapeutic index • tumor necrosis factor • vascular leak syndrome
\end{abstract}

\section{Introduction}

Interleukin 2 (IL-2) mediates the regression of certain human malignancies. Clinical trials demonstrate that response rates with IL-2 therapy for melanoma and renal cell carcinoma are comparable, or superior, to the best regimens of conventional chemotherapeutic agents (1). IL-2 therapy has, however, been associated with the development of dose-dependent systemic toxicities of sufficient severity to limit therapeutic efficacy and preclude clinical application for most patients (2). The IL-2induced toxicities of primary clinical significance occur second-

\footnotetext{
Address reprint requests to Dr. Edwards, Department of Surgery, University of Louisville, Louisville, KY 40292.

Received for publication 30 March 1992 and in revised form 11 May 1992.
}

J. Clin. Invest.

(c) The American Society for Clinical Investigation, Inc. 0021-9738/92/08/0637/05 \$2.00

Volume 90, August 1992, 637-641 ary to a "vascular leak syndrome" and include diffuse tissue edema, weight gain, decreased systemic vascular resistance with hypotension, acute respiratory distress, and hepatic and renal dysfunction.

The mechanism for the IL-2-induced vascular leak syndrome and related toxicities are not completely understood. However, they are remarkably similar to the systemic responses seen in severe infection or injury where tumor necrosis factor (TNF) ${ }^{1}$ is an important mediator (3). Previous studies also show that IL-2 induces macrophage production of TNF (4), and increased levels of plasma TNF have been measured in patients treated with IL-2 (5). In addition, Fraker et al. (6) demonstrated that passive immunization against TNF partially abrogated IL-2 toxicity in C57BL/6 mice, while the antitumor effect of IL-2 against pulmonary metastasis was maintained. Collectively, these studies suggest that the secondarily induced cytokine TNF is most likely a primary mediator of IL-2 toxicity, and that treatment strategies aimed to inhibit the production of TNF hold promise for extending the therapeutic index of IL-2. We hypothesized that one such treatment could involve the use of pentoxifylline (PTXF).

PTXF suppresses macrophage TNF gene transcription and increases the survival rate in murine endotoxic shock $(7,8)$. In addition, PTXF prevents IL-2-induced lung injury in guinea pigs (9) and also inhibits IL-2-induced leukocyte-endothelial adherence while reducing systemic toxicity (10). The present study tested the effects of PTXF on IL-2-induced systemic toxicities and on IL-2 antitumor efficacy.

The data of our investigation clearly show that PTXF suppresses the IL-2 induction of increased circulating levels of TNF as well as the IL-2 induction of lymphocyte infiltration in various organs while ameliorating IL-2 treatment toxicity. Most importantly, this inhibition of toxicity occurred while the antitumor efficacy of IL-2 was maintained.

\section{Methods}

Animals. Female C57BL/6 mice (18-20 g) were obtained from Charles Rivers Breeding Laboratories, Wilmington, MA. They were housed individually and fed standard laboratory chow and water ad lib. Approval for this project was obtained from the University of Louisville Animal Care and Use Committee and was in compliance with National Institute of Health guidelines.

1. Abbreviations used in this paper: LAK, lymphokine-activated killer; MCA, methylcholanthrene; PTXF, pentoxifylline; TNF, tumor necrosis factor. 
Materials. Purified recombinant IL-2 was a gift from HoffmanLaRoche, Inc., Nutley, NJ. The specific activity of the cytokine was 1.2 $\times 10^{7} \mathrm{U} / \mathrm{mg}$. Endotoxin contamination, measured by the Limulus amebocyte lysate assay, was $<0.312$ endotoxin U/10 $10^{6}$ IU IL-2. PTXF was donated by Hoechst-Roussell Pharmaceuticals, Inc. Both IL-2 and PTXF were diluted to the appropriate concentrations in sterile saline containing $1 \%$ homologous serum for intraperitoneal injection.

Transplantable tumor. A 3-methylcholanthrene-induced (MCA105) sarcoma (a gift from the laboratory of Dr. Raj K. Puri, Bethesda, MD) was transplanted into syngeneic C57BL/6 mice by subcutaneous injections of a tumor cell suspension. The developing subcutaneous sarcomas were excised when they reached $\sim 1.5 \mathrm{~cm}$ in diameter. The tumors were debrided of all tissue that appeared to be necrotic and nonsarcomatous. The remaining neoplastic tissue was then passed through a fine wire mesh, mincing the tissue into fragments $\sim 1 \mathrm{~mm}$ in size. Next, a single-cell suspension was obtained by digesting the remaining tumor fragments in $\mathbf{4 0 ~ m l}$ of Hank's balanced salt solution (HBSS) containing $100 \mathrm{U}$ of hyaluronidase, $40 \mathrm{mg}$ of collagenase, and $4 \mathrm{mg}$ of deoxyribonuclease (Sigma Chemical Co., St. Louis, MO) under constant stirring at room temperature for $3 \mathrm{~h}$. The cells were washed four times with HBSS, counted with a hemocytometer, and the concentration was adjusted to $\sim 1.5$ million cells/ml. For all experiments, the tumor suspension that was used for intravascular administration was from the fourth generation.

Experimental design. Female C57BL/6 mice were allowed to acclimate to their surroundings for $1 \mathrm{wk}$ before the initiation of the experiments. In the first series of experiments, nontumored C57BL/6 mice were randomly divided into four groups (Table I) and were injected with $0.1-\mathrm{ml}$ intraperitoneal injections as follows: The first group (IL-2 alone) received $100,000 \mathrm{U}$ of IL-2 every $6 \mathrm{~h}$ and saline every $12 \mathrm{~h}$. The second group (IL-2 and PTXF) was given 100,000 U of IL-2 every $6 \mathrm{~h}$ and $50 \mathrm{mg} / \mathrm{kg}$ PTXF every $12 \mathrm{~h}$. The third group (PTXF alone) was given $50 \mathrm{mg} / \mathrm{kg}$ of PTXF every $12 \mathrm{~h}$ and saline every $6 \mathrm{~h}$. The fourth group (controls) received an equal volume of saline every $6 \mathrm{~h}$. All animals were kept in the above protocol for $4 \mathrm{~d}$, receiving a total of 16 treatments.

$6 \mathrm{~h}$ after the last injection on day 5 , the mice were inspected and their appearance and activity level were graded and scored on the following morbidity scale: 1 , normal; 2 , decreased activity; 3 , ruffled fur, moved only with prompting; 4 , moribund; 5 , died.

Mice were then anesthetized with $50 \mathrm{mg} / \mathrm{kg}$ of pentobarbital, and blood was obtained for a complete blood count, serum glutamate pyruvate transaminase, serum glutamic-oxaloacetic transaminase, and determination of serum TNF level quantitated by an ELISA kit (Genzyme Corp., Cambridge, MA).

Samples of the lung, liver, jejunum, heart, pancreas, kidney, spleen, and thymus were excised. These samples were then fixed in $10 \%$ phosphate-buffered formaldehyde, dehydrated in graded alcohols and embedded in paraffin. At least eight sections of each organ from each animal were stained with hematoxylin and eosin. The identity of the tissue was coded, and the slides were randomly graded for lymphocytic infiltration. The remaining heart, liver, and splenic tissue, as well as a $3-\mathrm{cm}$ section of jejunum, one lung, and one kidney were then removed, weighed, and immediately frozen. They were next lyophilized at $-50^{\circ} \mathrm{C}$ for $24 \mathrm{~h}$ and weighed to determine the wet/dry weight ratio as a measurement of organ edema.

The second series of experiments evaluated the effects of IL-2 and PTXF in tumored female C57BL/6 mice. In these experiments mice were given mild pentobarbital anesthesia $(25 \mathrm{mg} / \mathrm{kg})$ by intraperitoneal injection, and $0.2 \mathrm{ml}$ of the tumor cell suspension $(\sim 300,000$ cells) was injected into their tail veins (6). $4 \mathrm{~d}$ after tumor cell injection, all animals were randomly divided into four groups (Table I). Mice were then treated for $4 \mathrm{~d}$ with combinations of either IL-2, PTXF, or saline, according to the dose and schedule outlined previously for the nontumored groups. $28 \mathrm{~d}$ after tumor cell injection, the mice were graded and scored on the morbidity scale and killed.

Thoracotomy was performed after pentobarbital overdose (100 $\mathrm{mg} / \mathrm{kg}$ ) and the lungs were injected with a solution comprising $15 \%$
India Ink and $1 \%$ ammonia via a small cannula placed in the trachea. The tumors were then "bleached" for $24 \mathrm{~h}$ in Fekete's solution containing $100 \mathrm{ml}$ of $70 \%$ alcohol, $10 \mathrm{ml}$ of formaldehyde, and $5 \mathrm{ml}$ of glacial acetic acid (6). Pulmonary metastases appeared as white on a black background of normal pulmonary parenchyma. Metastases were then counted under a dissecting microscope to assess tumor burden.

In addition to counting the number of metastases, an image analysis system was used to generate an index of tumor surface area. Lungs were viewed through a closed circuit television system and the image of each side of each lung was digitized using a PC Vision Plus (Imaging Technology, Woburn, MA) digitizer with Image Pro Plus (Media Cybernetics, Silver Spring, MD) software. The digitized images consisted of $\sim 130,000$ pixels of varying gray levels. The white tumors gave high gray level values, and the black normal pulmonary parenchyma gave low gray level values. The average gray level above normal pulmonary parenchymal background for each side of each lung was determined, and values from the two sides of each lung were averaged.

Statistical analyses. Statistical analysis was performed by analysis of variance with Tukey-Kramer honestly significant difference. Differences were considered significantly different at $P \leq 0.05$.

\section{Results}

IL-2 induced significant morbidity and mortality in both nontumored mice and tumored mice (Table I). 3 of 13 (23\%) nontumored mice died after treatment with IL-2 alone. In addition, significant morbidity was evident by lethargy and ruffled fur in nontumored mice that were given IL-2 alone as compared with the other three groups of nontumored mice. Similarly, 3 of $10(30 \%)$ tumored mice that received IL-2 alone died by the completion of IL-2 treatment (Table I). At this time, tumored mice had a similar magnitude of increased treatment morbidity (IL-2 toxicity) as compared to nontumored mice (Table I).

Of the seven tumored mice that survived $4 \mathrm{~d}$ of IL- 2 treatment, all were alive at the end of the 28 -d experiment. There were also no deaths in the group of tumored mice that were treated with a combination of IL- 2 and PTXF during the entire experiment. However, a total of five $(43 \%)$ tumored mice not receiving IL-2 died before the end of the experiment, presumably of progressive tumor burden. These included three $(50 \%)$ mice from the control group and two (33\%) from the PTXF alone group. The mice that survived to the end of the experiment experienced significantly greater morbidity (graded at day 28) than animals in either of the groups treated with IL-2 (Table I).

There was no difference in the histological findings of nontumored mice treated with either PTXF alone or the saline control. However, significant histological differences existed between the controls and animals treated with IL-2 alone (Table II). Nontumored mice that were treated with IL-2 alone had a marked degree of lymphocytic infiltration of the vascular walls and perivascular spaces of venules, capillaries, and small arterioles in the lung, liver, pancreas, and small bowel mesentery (Table II). Pulmonary alveolar septa were markedly thickened and alveoli frequently contained erythrocytes, macrophages, and abundant lymphocytes. There was a marked lymphocytic infiltration of hepatic portal triads and central venular areas and occasional areas of hepatic necrosis. The heart was normal except for a few lymphoid cells in the epicardium.

Significantly less lymphocytic infiltration was seen in the lung, liver, pancreas, and small bowel mesentery in the IL-2 and PTXF group as compared to mice treated with IL-2 alone. 


\begin{tabular}{|c|c|c|c|c|}
\hline & \multicolumn{4}{|c|}{ Experimental groups } \\
\hline & Control & PTXF & IL-2 & IL-2 and PTXF \\
\hline \multicolumn{5}{|l|}{ Nontumored mice } \\
\hline Number of mice $(n)$ & 10 & 10 & 13 & 10 \\
\hline Treatment mortality* $(\%)$ & 0 & 0 & 23 & 0 \\
\hline Treatment morbidity score* & $1.1 \pm 0.1$ & $1.1 \pm 0.1$ & $3.2 \pm 0.4^{\prime \prime}$ & $1.3 \pm 0.1^{1}$ \\
\hline \multicolumn{5}{|l|}{ Tumored mice } \\
\hline Number of mice $(n)$ & 6 & 6 & 10 & 6 \\
\hline Treatment mortality ${ }^{\ddagger}(\%)$ & 0 & 0 & 30 & 0 \\
\hline Treatment morbidity score ${ }^{\ddagger}$ & $1.2 \pm 0.1$ & $1.0 \pm 0.0$ & $3.3 \pm 0.4^{\prime \prime}$ & $1.6 \pm 0.2^{1}$ \\
\hline Tumor-related mortality $(\%)$ & 50 & 33 & 0 & 0 \\
\hline Tumor-related morbidity score & $4.0 \pm 0.3$ & $3.7 \pm 0.4$ & $1.1 \pm 0.1^{\prime \prime}$ & $1.1 \pm 0.1^{11}$ \\
\hline
\end{tabular}

All data expressed as mean \pm SE. * Data taken the day after completion of $4 \mathrm{~d}$ of treatment. ${ }^{\ddagger}$ Data taken the day after completion of $4 \mathrm{~d}$ of treatment ( $9 \mathrm{~d}$ after tail vein injection of tumor cell suspension). ${ }^{8}$ Data taken $28 \mathrm{~d}$ after tail vein injection of tumor cell suspension. $P \leq 0.05$ by ANOVA with Tukey-Kramer honestly significant difference: (") compared with the control group and (") compares the IL-2 group with the IL-2 and PTXF group.

However, the group receiving both IL-2 and PTXF did have more lymphocytes present than controls. In contrast to the IL-2-induced lymphocytic infiltration observed in most organs, there was significant depletion of thymic lymphocytes in mice treated with either IL-2 alone or with a combination of IL-2 and PTXF (Table II).

There was no significant difference in multiple organ edema (wet/dry weight ratios) in mice treated with either saline or PTXF (Table II), except for the spleen where significantly less edema was observed in mice treated with PTXF. Mice treated with IL-2 alone had increased edema in the lung, liver, jejunum, and spleen. The addition of PTXF prevented IL-2induced edema in these organs.

IL-2 significantly increased the circulating serum level of TNF (Table II). The combination of PTXF and IL-2 significantly suppressed IL-2-induced increases in circulating TNF. PTXF also increased the number of circulating white blood cells and lymphocytes as compared to controls (Table II), presumably because of the inhibition of leukocyte margination and adhesion. IL-2 caused leukopenia, lymphopenia, and

Table II. Toxicity in Nontumored Mice

\begin{tabular}{|c|c|c|c|c|}
\hline & \multicolumn{4}{|c|}{ Experimental groups } \\
\hline & Controls & PTXF & IL-2 & IL-2 and PTXF \\
\hline \multicolumn{5}{|l|}{ Lymphocytic infiltration } \\
\hline Lung & $1.1 \pm 0.1$ & $1.0 \pm 0.0$ & $5.0 \pm 0.0^{*}$ & $2.2 \pm 0.3^{* \pm}$ \\
\hline Liver & $1.0 \pm 0.0$ & $1.0 \pm 0.0$ & $4.8 \pm 0.1^{*}$ & $2.0 \pm 0.2^{* \pm}$ \\
\hline Jejunal mesentery & $1.1 \pm 0.1$ & $1.0 \pm 0.0$ & $4.4 \pm 0.3^{*}$ & $2.4 \pm 0.3^{* \ddagger}$ \\
\hline Heart & $1.0 \pm 0.0$ & $1.0 \pm 0.0$ & $1.3 \pm 0.2$ & $1.0 \pm 0.0$ \\
\hline Pancreas & $1.1 \pm 0.0$ & $1.1 \pm 0.1$ & $4.3 \pm 0.3^{*}$ & $2.0 \pm 0.3^{* \ddagger}$ \\
\hline Thymus & $5.0 \pm 0.0$ & $4.7 \pm 0.2$ & $2.7 \pm 0.2^{*}$ & $3.1 \pm 0.2^{*}$ \\
\hline \multicolumn{5}{|l|}{ Organ wet/dry weight ratios } \\
\hline Lung & $4.0 \pm 0.1$ & $3.9 \pm 0.1$ & $5.0 \pm 0.1^{*}$ & $4.2 \pm 0.1^{\ddagger}$ \\
\hline Liver & $3.5 \pm 0.1$ & $3.4 \pm 0.1$ & $4.0 \pm 0.1^{*}$ & $3.7 \pm 0.1^{\ddagger}$ \\
\hline Jejunum & $4.0 \pm 0.1$ & $3.6 \pm 0.1$ & $4.6 \pm 0.1^{*}$ & $3.9 \pm 0.1^{\ddagger}$ \\
\hline Heart & $4.0 \pm 0.2$ & $4.1 \pm 0.1$ & $4.0 \pm 0.1$ & $4.1 \pm 0.1$ \\
\hline Kidney & $3.6 \pm 0.1$ & $3.5 \pm 0.1$ & $4.0 \pm 0.1$ & $3.7 \pm 0.1$ \\
\hline Spleen & $4.2 \pm 0.1$ & $3.7 \pm 0.1^{*}$ & $5.0 \pm 0.1^{*}$ & $4.4 \pm 0.1^{\ddagger}$ \\
\hline \multicolumn{5}{|l|}{ Serum laboratory test } \\
\hline $\operatorname{TNF}(n g / m l)$ & $0.06 \pm 0.05$ & $0.32 \pm 0.02$ & $1.04 \pm 0.11^{*}$ & $0.31 \pm 0.05^{\ddagger}$ \\
\hline $\mathrm{WBC}(\times 1,000)$ & $4.0 \pm 0.2$ & $7.0 \pm 0.3^{*}$ & $1.5 \pm 0.1^{*}$ & $11.2 \pm 1.7^{* \pm}$ \\
\hline Total lymphocytes $(\times 1,000)$ & $3.4 \pm 0.2$ & $6.3 \pm 0.3^{*}$ & $0.7 \pm 0.1^{*}$ & $10.7 \pm 1.7^{* \pm}$ \\
\hline Platelets $(\times 1,000)$ & $773 \pm 35$ & $805 \pm 16$ & $544 \pm 23^{*}$ & $750 \pm 29^{\ddagger}$ \\
\hline Serum glutamate pyruvate transaminase & $27 \pm 3$ & $23 \pm 6$ & $104 \pm 8^{*}$ & $38 \pm 7^{\ddagger}$ \\
\hline Serum glutamic-oxaloacetic transaminase & $130 \pm 8$ & $153 \pm 29$ & $353 \pm 28^{*}$ & $162 \pm 28^{\ddagger}$ \\
\hline
\end{tabular}

All data expressed as mean \pm SE. $P \leq 0.05$ by ANOVA with Tukey-Kramer HSD: $\left({ }^{*}\right)$ compared with the control group and $\left({ }^{\ddagger}\right)$ compares the IL-2 group to the IL-2 and PTXF group. 
thrombocytopenia $4 \mathrm{~d}$ after therapy. The addition of PTXF to IL-2 therapy resulted in a lymphophilia as compared to controls. The IL-2-induced leukopenia and thrombocytopenia were also blocked by PTXF. IL-2 induced hepatic dysfunction manifested by increased serum transaminase levels, and PTXF inhibited the IL-2-induced hepatic dysfunction but had no effect on liver function by itself.

Tail vein injections of a tumor cell suspension of MCA-105 fibrosarcoma cells resulted in a large number of pulmonary metastases both in the control group and the group treated with PTXF alone. The number of metastases and the index of tumor surface area were significantly reduced by IL- 2 treatment (Table III). The addition of PTXF to IL-2 therapy did not alter the antitumor efficacy of IL-2 (Table III and Fig. 1).

\section{Discussion}

In these studies, PTXF did not alter the antitumor efficacy of IL-2 but it did inhibit many of the indices of IL-2 toxicity. PTXF suppressed IL-2-induced elevation of circulating TNF, it decreased IL-2-induced lymphocytic infiltration, and it decreased IL-2-induced multiple organ edema. It also inhibited IL-2-induced increases in circulating liver enzymes and it decreased IL-2-induced morbidity and mortality. These toxic effects of IL-2 are consistent with previous reports (2) and are the current primary limitation to the clinical application of IL-2 as an effective antineoplastic agent. Several studies have evaluated the mechanism of the vascular leak syndrome and identified potential inhibitors (2), but, to date, no clinically approved drugs have been identified that reduce toxicity and at the same time preserve antitumor efficacy. Our study identifies PTXF as an agent with the potential for extending the therapeutic efficacy of IL-2.

In vivo evidence shows that IL-2 induces the production of other cytokines that are potentially important in mediating IL2 toxicities. IL-2 induces macrophage TNF gene expression in vivo $(4,11)$, and elevated circulating levels of TNF have been detected in patients treated with IL-2 $(5,12)$. Mier et al. (5) have also demonstrated that patients treated with IL-2 have increased circulating levels of TNF temporally consistent with the onset of fever and other toxic manifestations of IL-2.

The current study also demonstrated elevated plasma levels of TNF after IL-2 therapy (Table II). IL-2 has no direct effect on endothelial permeability (13), but TNF induces acute pulmonary vascular endothelial injury and increases vascular permeability $(14,15)$. Skin biopsies of patients treated with IL-2 demonstrate the expression of endothelial leukocyte adhesion molecules (16). It is TNF, and not IL-2, that induces the ex-

Table III. Tumor Response

\begin{tabular}{lcccc}
\hline & \multicolumn{4}{c}{ Experimental groups } \\
\cline { 2 - 5 } \multicolumn{1}{c}{ Tumor response } & Controls & PTXF & IL-2 & IL-2 and PTXF \\
\hline $\begin{array}{l}\text { Number of metastases/ } \\
\text { lung }\end{array}$ & $114 \pm 9$ & $121 \pm 12$ & $6 \pm 3^{\ddagger}$ & $11 \pm 4^{\ddagger}$ \\
Index of tumor area* & $18.6 \pm 0.9$ & $21.1 \pm 1.8$ & $2.2 \pm 0.8^{\ddagger}$ & $1.8 \pm 1.0^{\ddagger}$ \\
\hline
\end{tabular}

${ }^{\ddagger} P \leq 0.05$ by ANOVA with Tukey-Kramer HSD compared with the control group. * Image analysis technique giving mean $\pm \mathrm{SE}$ gray level of lungs. pression of endothelial leukocyte adhesion molecules. In addition, neither nude or irradiated mice treated with IL-2 develop the vascular leak syndrome (17). Collectively, these observations suggest that IL-2 induces the production and release of TNF, which may then mediate the vascular leak syndrome and related toxicities of IL-2 through interactions between activated immune effector cells and the microvascular endothelium.

Several recent reports have documented the PTXF-mediated suppression of TNF production $(7,8)$, but, to the best of our knowledge, the current report is the first to correlate the PTXF attenuation of IL-2-induced toxicity with the synchronous PTXF suppression of circulating plasma levels of TNF. Most importantly, despite the PTXF inhibition of IL-2 toxicity, IL-2 antitumor efficacy was still present with PTXF treatment (Table III). This suggests that while TNF is a probable cause of systemic toxicity of IL-2, TNF may not be involved in the antitumor efficacy of IL-2.

In addition to TNF, lymphokine-activated killer (LAK) cells are known to be important in the genesis of IL-2 toxicity as well as in mediating the antitumor efficacy of IL-2. 3-4 d after treatment with IL-2, lymphocytes are activated and capable of tumor cell lysis (18). Damle et al. (19) have shown in vitro that LAK cells exhibit enhanced adhesion to normal vascular endothelial cells and cause their lysis. Selective depletion of one subset of IL-2-activated lymphocytes (those bearing the asiolo-GM1 surface glycolipid) prevented the toxicity seen $5 \mathrm{~d}$ after IL-2 administration (20). These findings strongly suggest that the mechanism of the vascular leak syndrome involves LAK cells.

Our study extends the findings of these previous reports in showing that mice treated with IL-2 alone had changes in lymphocyte distribution (Table II) that correlated with the development of toxicity (Tables I and II). IL-2 therapy resulted in the depletion of thymic lymphocytes, the development of peripheral lymphopenia, and the formation of lymphoid infiltrates in the lung, liver, small bowel mesentery, and pancreas.

PTXF remarkably altered the distribution of IL-2-activated lymphocytes but did not interfere with the IL-2-induced mobilization of lymphocytes, in that mice treated with IL-2 alone and those treated with IL-2 and PTXF both had similar degrees of thymic depletion (Table II). Mice treated with a combination of IL-2 and PTXF had much larger numbers of circulating lymphocytes in peripheral blood than mice treated with IL-2 alone, but they had a marked decrease in the number of lymphocytes infiltrating into the parenchyma of various organs (Table I). We believe that the current report is also the first observation of the PTXF suppression of IL-2-induced lymphocyte trafficking from the intravascular space to the parenchyma of various organs. The resultant reduction in lymphocytic infiltration in various organs may be another mechanism in the PTXF prevention of multiple organ edema, dysfunction, and associated morbidity and mortality of IL-2 therapy.

Despite this PTXF-mediated redistribution of lymphocytes, IL-2 still maintained its antitumor efficacy (Fig. 1 and Table III). Sasaki et al. (21) have shown that in the presence of IL-2, LAK cells demonstrate an increased frequency of longterm adhesive interactions within the tumor microvasculature compared with the normal microcirculation. Perhaps by preventing the lymphocytic infiltration of normal tissues, and thus maintaining an increased number of circulating LAK cells, PTXF provides for a greater number of LAK cells available for 


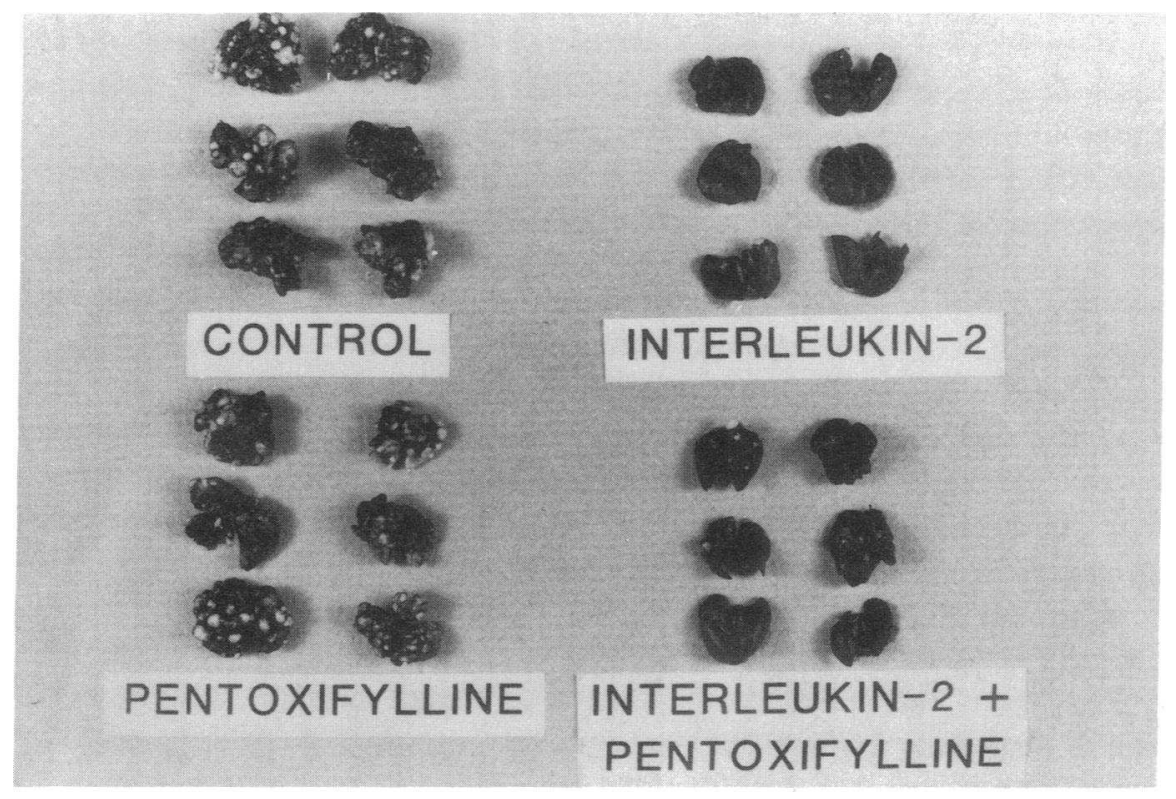

Figure 1. Antitumor efficacy. Lungs harvested from mice $28 \mathrm{~d}$ after tail vein injection of $3 \times 10^{5}$ MCA-105 fibrosarcoma cells. 4 d after tumor cell injection mice were treated with 16 intraperitoneal injections of combinations of either IL-2 $\left(1 \times 10^{5} \mathrm{U}\right.$ given every $6 \mathrm{~h})$, PTXF ( $50 \mathrm{mg} / \mathrm{kg}$ given every $12 \mathrm{~h}$ ), or equal volumes of saline. Lungs were treated with India ink and Fekete's solution such that tumor metastases appear white on a black background of normal pulmonary parenchyma. There were significantly fewer tumors in the lungs of animals treated with IL2 (top right) when compared to controls (top left). No antitumor efficacy was seen with PTXF alone (bottom left). PTXF did not alter the antitumor efficacy of IL-2 (bottom right).

interaction within the tumor microcirculation, thus maintaining IL-2 antitumor efficacy.

\section{Acknowledgments}

This study was supported by American Cancer Society Clinical Oncology Career Development Award (CDA-91-178), Atlanta, GA, to Dr. Edwards.

\section{References}

1. Rosenberg, S. A., M. T. Lotze, J. C. Yang, P. M. Aebersold, W. M. Linehan, C. A. Seipp, and D. E. White. 1989. Experience with the use of high-dose interleukin-2 in the treatment of 652 cancer patietns. Ann. Surg. 210:474-485.

2. Siegel, J. P., and R. K. Puri. 1991. Interleukin-2 toxicity. J. Clin. Oncol. 9:694-704.

3. Starnes, H. F., R. S. Warren, M. Jeevanandam, J. L. Gibrilove, W. Larchian, H. F. Oettgen, and M. F. Brennan. 1988. Tumor necrosis factor and the acute metabolic response to tissue injury in man. J. Clin. Invest. 82:1321-1325.

4. Remick, D. G., D. T. Nguyen, M. K. Eskandari, and S. L. Kunkel. 1991 Interleukin 2 induces tumor necrosis factor gene expression in vivo. Immunol. Invest. 20:395-405.

5. Mier, J. W., G. Vachino, J. W. M. Van Der Meer, R. P. Numerof, S. Adams, J. G. Cannon, H. A. Bernheim, M. B. Atkins, D. R. Parkinson, and C. A Dinarello. 1988. Induction of circulating tumor necrosis factor (TNF $\alpha$ ) as the mechanism for the febrile response to interleukin-2 (IL-2) in cancer patients. $J$. Clin. Immunol. 8:426-436.

6. Fraker, D. L., H. N. Langstein, and J. A. Norton. 1989. Passive immunization against tumor necrosis factor partially abrogates interleukin 2 toxicity. $J$. Exp. Med. 170:1015-1020.

7. Strieter, R. M., D. G. Remick, P. A. Ward, R. N. Spengler, J. P. Lynch, J. Larrick, and S. L. Kunkel. 1988. Cellular and molecular regulation of tumor necrosis factor-alpha production by pentoxifylline. Biochem. Biophys. Res. Commun. 155:1230-1236.

8. Schade, U. F. 1990. Pentoxifylline increases survival in murine endotoxin shock and decreases formation of tumor necrosis factor. Circ. Shock. 31:171-181.

9. Ishizaka, A., J. R. Hatherill, H. Harada, M. Yonemaru, H. Hoffmann, H. Zheng, P. T. O'Hanley, and T. A. Raffin. 1989. Prevention of interleukin-2-in- duced acute lung injury in guinea pigs by pentoxifylline. J. Appl. Physiol. 67:2432-2437.

10. Edwards, M. J., D. L. Abney, and F. N. Miller. 1991. Pentoxifylline inhibits interleukin-2-induced leukocyte-endothelial adherence and reduces systemic toxicity. Surgery. 110:199-204.

11. Doherty, G. M., J. C. Jensen, H. R. Alexander, C. M. Buresh, and J. A. Norton. 1991. Pentoxifylline suppression of tumor necrosis factor gene transcription. Surgery. 110:192-198.

12. Gemlo, B. T., M. A. Palladino, H. S. Jaffe, T. P. Espevik, and A. A. Rayner. 1988. Circulating cytokines in patients with metastatic cancer treated with recombinant interleukin 2 and lymphokine-activated killer cells. Cancer Res. 48:5864-5867.

13. Damle, N. K., and L. V. Doyle. 1989. IL-2-activated human killer lymphocytes but not their secreted products mediate increase in albumin flux across cultured endothelial monolayers. J. Immunol. 142:2660-2669.

14. Goldblum, S. E., B. Hennig, M. Jay, K. Yoneda, and C. J. McClain. 1989. Tumor necrosis factor $\alpha$-induced pulmonary vascular endothelial injury. Infect. Immun. 57:1218-1226.

15. Larrick, J. W., and S. C. Wright. 1990. Cytotoxic mechanisms of tumor necrosis factor- $\alpha$. FASEB (Fed. Am. Soc. Exp. Biol.) J. 4:3215-3223.

16. Cotran, R. S., J. S. Pober, M. A. Gimbrone, T. A. Springer, E. A. Wiebke, A. A. Gaspari, S. A. Rosenberg, and M. T. Lotze. 1987. Endothelial activation during interleukin 2 immunotherapy: a possible mechanism for the vascular leak syndrome. J. Immunol. 139:1883-1888.

17. Rosenstein, M., S. E. Ettinghausen, and S. A. Rosenberg. 1986. Extravasation of intravascular fluid mediated by the systemic administration of recombinant interleukin 2. J. Immunol. 137:1735-1742.

18. Lotze, M. T., Y. L. Matory, S. E. Ettinghausen, A. A. Rayner, S. O. Sharrow, C. A. Y. Seipp, M. C. Custer, and S. A. Rosenberg. 1985. In vivo administration of purified human interleukin-2. II. Half-life, immunologic effects and expansion of peripheral lymphoid cells in vivo with recombinant IL-2. J. Immunol. 135:2865-2875.

19. Damle, N. K., L. V. Doyle, J. R. Bender, and E. C. Bradley. 1987. Interleukin-2 activated human lymphocytes exhibit enhanced adhesion of normal vascular endothelial cells and cause their lysis. J. Immunol. 138:1779-1785.

20. Anderson, T. D., T. J. Hayes, M. K. Gately, J. M. Bontempo, L. L. Stern, and G. A. Truitt. 1988. Toxicity of human recombinant interleukin-2 in the mouse is mediated by interleukin-activated lymphocytes: separation of efficacy and toxicity by selective lymphocyte subset depletion. Lab. Invest. 59:598-612.

21. Sasaki A., R. J. Melder, T. L. Whiteside, R. B. Herberman, and R. K. Jain. 1991. Preferential localization of human adherent lymphokine-activated killer cells in tumor microcirculation. J. Natl. Cancer Inst. 83:433-437. 